\title{
Mechanical and biocompatible properties of polymer-infiltrated- ceramic-network materials for dental restoration
}

\author{
Bencang CUI ${ }^{a}$, Ranran $\mathrm{ZHANG}^{a}$, Fengbo $\mathrm{SUN}^{a}$, Qian $\mathrm{DING}^{b}$, \\ Yuanhua LIN ${ }^{a, *}$, Lei ZHANG ${ }^{b}$, Cewen NAN ${ }^{a}$ \\ ${ }^{a}$ School of Materials Science and Engineering, Tsinghua University, Beijing 100084, China \\ ${ }^{b}$ Department of Prosthodontics, School and Hospital of Stomatology, Peking University, Beijing 100081, China
}

Received: April 11, 2019; Revised: May 26, 2019; Accepted: June 8, 2019

(c) The Author(s) 2019.

\begin{abstract}
Dental restorative materials with high mechanical properties and biocompatible performances are promising. In this work, polymer-infiltrated-ceramic-network materials (PICNs) were fabricated via infiltrating polymerizable monomers into porous ceramic networks and incorporated with hydroxyapatite nano-powders. Our results revealed that the flexural strength can be enhanced up to 157.32 $\mathrm{MPa}$, and elastic modulus and Vickers hardness can be achieved up to 19.4 and $1.31 \mathrm{GPa}$, respectively, which are comparable with the commercial computer-aided design and computer-aided manufacturing (CAD/CAM) blocks. Additionally, the adhesion and spreading of rat bone marrow mesenchymal stem cells (rBMSCs) on the surface of such materials can be improved by adding hydroxyapatite, which results in good biocompatibility. Such PICNs are potential applicants for their application in the dental restoration.
\end{abstract}

Keywords: dental restoration; hydroxyapatite; polymer-infiltrated-ceramic-network materials (PICNs); mechanical property; biocompatible performance

\section{Introduction}

The developments of computer-aided design and computer-aided manufacturing (CAD/CAM) systems have contributed in the increasing usage of $\mathrm{CAD} / \mathrm{CAM}$ blocks. Composites, combining the properties of ceramics and polymers, have the potential to manipulate the mechanical performances. Especially, the development of polymer-infiltrated-ceramic-network materials (PICNs), inspired by the glass infiltrated ceramic, marked great progress in imitating the mechanical properties of natural dentin and enamel [1,2]. PICNs are different

* Corresponding author.

E-mail: linyh@tsinghua.edu.cn from filled composites because the polymer is a continuous phase in the filled composites and the ceramic particles are dispersed in the polymer matrix. PICNs were fabricated by infiltrating the polymerizable monomers into partially sintered porous ceramics and curing by heat induced polymerization. This possesses two interpenetrating networks, i.e., polymer and ceramic phases.

PICNs have been discussed as typical dental restorative materials since the commercial launch of VITA ENAMIC by VITA Zahnfabrik in 2012 [1]. These materials contain many characteristics. PICNs have shown much higher Weibull modulus of flexural strength than ceramics and other types of dental composites, indicating their minor strength variations [3]. Although the hardness of 
PICNs was lower than that of ceramics, the material loss values after artificial chewing cycles can be comparable, if it is not less than that of the ceramics [3]. PICNs also exhibit less strength reduction than dental ceramics after adjustment machining procedures. Color stainability of PICNs was found similar to some ceramic blocks and higher than that of filled composites [4,5].

Biocompatibility is also a significant property of dental materials in addition to mechanical and aesthetic properties. Hydroxyapatite possesses chemical compositions and mineral structures similar to that of the natural tooth and bone. Therefore, it is being used frequently as biomaterials or effective modifiers to improve the biocompatibilities of other materials. Hydroxyapatite has been used in filled resin composite many times. However, few studies were reported for its use in PICNs so far. The addition of hydroxyapatite may both affect the mechanical properties and biocompatibility of PICNs. In this work, our results indicate that the addition of hydroxyapatite in PICNs exhibits high mechanical properties and good biocompatibilities.

\section{Experimental}

The raw materials used in this study include sodium aluminum silicate (SIPERNAT 820A, Degussa AG, Germany), Bis-GMA, TEGDMA (Aladdin Reagents Company), and Dibenzoylperoxide (BPO, J \& K Scientific Ltd.).

In order to modify the biocompatibility of this PICN, nano-hydroxyapatite was mixed into this PICN. Concentrations of $1,2,3$, and $4 \mathrm{wt} \%$ (referring to hydroxyapatite/porous ceramics in this study) of nanohydroxyapatite powders were mixed into sodium aluminum silicate via ballmilling. The ball milling process lasted for $24 \mathrm{~h}$ at a speed of $300 \mathrm{rpm}$. Deionized water was used as the mixing medium. The slurry was dried in a drying oven at a temperature of $70{ }^{\circ} \mathrm{C}$. Powders without hydroxyapatite were used as the control group. Powders were poured into a stainless steel mold and compressed into blocks with the pressure of $3 \mathrm{MPa}$ and hold for 3 min followed by an isostatic cool pressure of $220 \mathrm{MPa}$. Blocks were removed in a mid-temperature furnace and sintered up to $700{ }^{\circ} \mathrm{C}$ at a heating rate of $5{ }^{\circ} \mathrm{C} / \mathrm{min}$ and preservation time of $2 \mathrm{~h}$, followed by a natural cooling process at 20-25 ${ }^{\circ} \mathrm{C}$. Porous ceramic blocks were treated with a silane coupling agent 3-(trimethoxysilyl) propyl methacrylate, i.e., $\gamma$-MPS (Aldrich Chemical Co.) prior to monomers infiltration to increase the chemical combination between ceramic and polymer. Monomer compounds containing Bis-GMA (49.5 wt $\%$ ), TEGDMA $(49.5 \mathrm{wt} \%)$, and BPO (1 wt $\%)$ were infiltrated into porous ceramic blocks via capillary action in a vacuum oven. After systematic infiltration of monomers, a polymerization process was induced by heating in a drying oven at $70{ }^{\circ} \mathrm{C}$ for $8 \mathrm{~h}$ and $110{ }^{\circ} \mathrm{C}$ for $8 \mathrm{~h}$.

The obtained PICNs samples were scrubbed to a thickness of $2 \pm 0.1 \mathrm{~mm}$. Long bars of $(2 \pm 0.1) \mathrm{mm} \times$ $(2 \pm 0.1) \mathrm{mm} \times 15 \mathrm{~mm}$ were cut from corresponding composite blocks and then polished systematically. The testing of flexural strength was carried out using a universal test machine (Shimadzu, EZ-100, Japan) in a three-point bending format (according to EN ISO-4049) with a constant loading speed of $0.75 \mathrm{~mm} / \mathrm{min}$ [6]. The maximum stress at fracture $\left(\sigma_{\mathrm{f}}\right)$ was calculated by Eq. (1):

$$
\sigma_{\mathrm{f}}=\frac{3 F l}{2 b d^{2}}
$$

where $F$ is the maximum load value at fracture; $l$ is the distance between supports (here $l=10 \mathrm{~mm}$ ); $b$ is the width (here $b=2 \mathrm{~mm}$ ); and $d$ is the height (here $d=$ $2 \mathrm{~mm}$ ) of the specimen. The values of $b$ and $d$ were measured at the center of each bending bar.

Ten nano-indentations were performed for each material with a fixed depth of $1000 \mathrm{~nm}$ using a nanoindentation tester (MTS, Keysight, G200, USA). Values of Vickers hardness and elastic modulus were calculated from nano-indentation results according to a method reported by Oliver and Pharr [7].

The samples (6 samples for each material) were sterilized at high temperature and high pressure in a sterilizing pot and then transferred into 96-well plates with 10,000 cells per well for subsequent detection. The cell viability was measured by the CCK- 8 methods at a cultural time of 1 day, 3 days, and 5 days. The cells were also cultured for 1 day, 3 days, and 5 days. Then the culture medium was sucked out and washed once with PBS for $5 \mathrm{~min} .150 \mu \mathrm{L}$ of complete medium containing 10\% CCK- 8 reagent was added into corresponding wells and cultured in an incubator for $2 \mathrm{~h}$ at $37{ }^{\circ} \mathrm{C}$. After that, the culture medium containing $10 \%$ CCK-8 reagent was transferred into a new $96-w e l l$ plate. New complete medium was added to the 96-well plate containing the cultured cells. The cells were cultured in an incubator at $37{ }^{\circ} \mathrm{C}$ for further examination. The absorbance value of the above solution containing $10 \%$ CCK- 8 reagent was detected 
by an enzyme labeling instrument (EnSpire, PerkinElmer, USA) at a wavelength of $450 \mathrm{~nm}$. One-way ANOVA tests were used for the statistical analysis ( $p=0.05$ was set as the significance level).

After 4 days of culture, the cells were fixed with $2.5 \%$ glutaraldehyde and placed in a refrigerator $4{ }^{\circ} \mathrm{C}$ temperature for $12 \mathrm{~h}$. The glutaraldehyde solution was sucked out and washed three times with PBS solution for $5 \mathrm{~min}$ each time. The gradient dehydration of ethanol aqueous solution and tert-butanol ethanol solution was carried out. In ethanol aqueous solution, the volume fraction of ethanol is $30 \%, 50 \%, 75 \%, 80 \%, 90 \%, 95 \%$, and $100 \%$. In a tert-butanol ethanol solution, the volume fraction of tert-butanol was $25 \%, 50 \%, 75 \%$, and $100 \%$. The cell samples were dehydrated twice at each concentration, each time for $10 \mathrm{~min}$. Then, $150 \mu \mathrm{L}$ of pure tert-butanol was added to each well and frozen in a refrigerator at $4{ }^{\circ} \mathrm{C}$. The samples were freeze-dried by a freeze-drying machine. The surface of the sample was treated with platinum spray and the morphology of PICNs and the cells were observed by scanning electron microscope (SEM).

\section{Results and discussion}

Figure 1 shows the structure and morphology of hy-
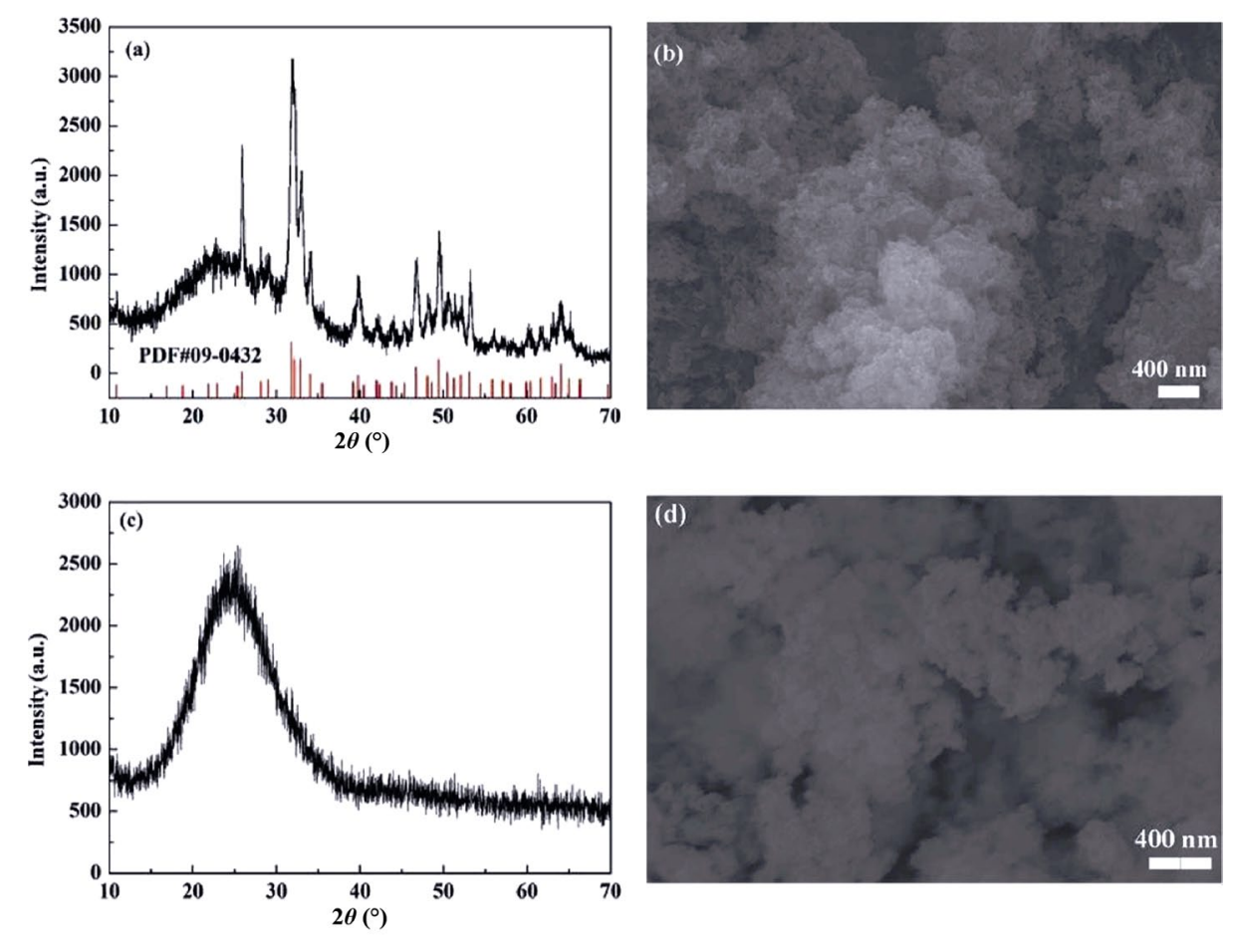

Fig. 1 Structure and morphology of hydroxyapatite and sodium aluminum silicate: (a, b) hydroxyapatite powder and (c, d) sodium aluminum silicate powder.

droxyapatite and sodium aluminum silicate. Hydroxyapatite possesses a crystalline structure, in line with PDF\# 09-0432. A glass phase is indicated in Fig. 1(c) for sodium aluminum silicate powder. Both powders appear to be in the form of nano-clusters.

Figure 2 represents the SEM observation of the fabricated PICNs. Two kinds of interpenetrating phases (ceramic and polymer) can be observed from them. No phase separations and buried cracks were found, indicating systematic infiltration of the polymers and the complete combination of the two phases. As shown in Fig. 2(b), it can be observed that the addition of hydroxyapatite has an effect on the fracture morphology.

For dental restorative composites, essential properties are mutually restricted, including mechanical properties, aesthetic properties, biocompatibility, etc. Chemical and structural modifications designed for the improvement of one kind of performance can be accompanied by a decrease in other properties. The mechanical properties (flexural strength, elastic modulus, and fracture toughness) of PICNs with hydroxyapatite are shown in Fig. 3.

The flexural strength varies from $82.19 \pm 13.88$ to $157.32 \pm 12.69 \mathrm{MPa}$. With the increase of hydroxyapatite content, the flexural strength of PICN shows a general decreasing trend. The flexural strength values were found very similar to that of commercial CAD/ 


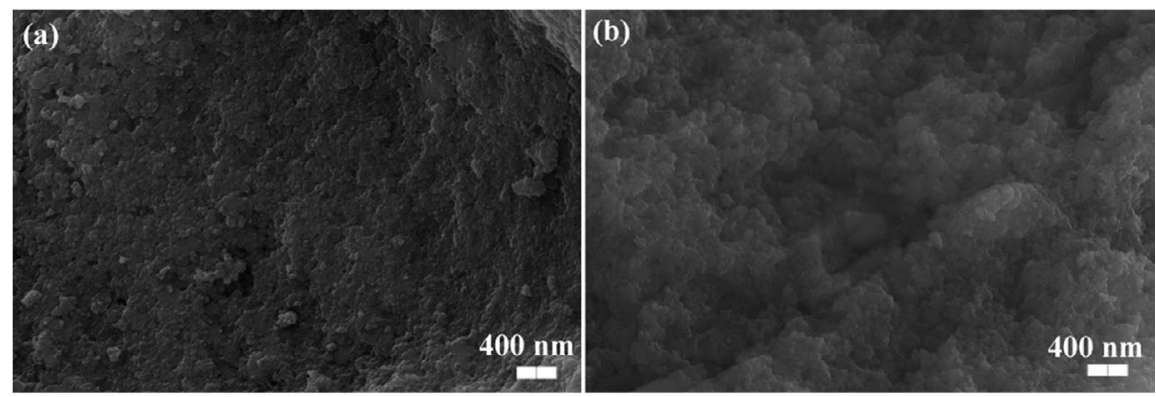

Fig. 2 SEM observations of PICNs: (a) PICN without hydroxyapatitea and (b) PICN with $2 \mathrm{wt} \%$ hydroxyapatite.
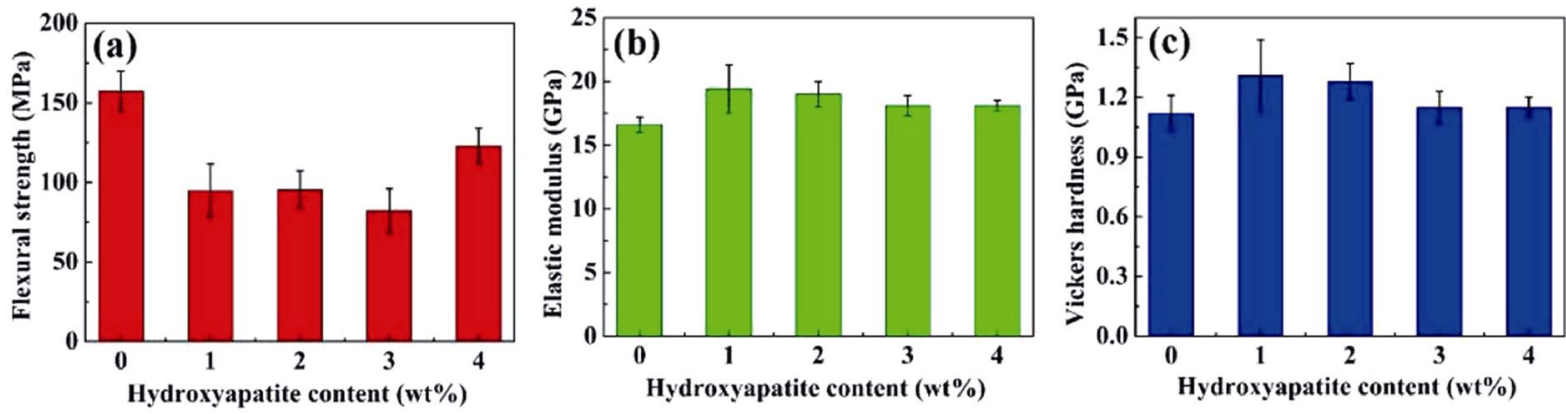

Fig. 3 Effect of hydroxyapatite addition on the mechanical properties of PICNs: (a) flexural strength, (b) elastic modulus, and (c) Vickers hardness.

CAM block Vita Enamic [8,9]. The flexural strength decreases significantly with the addition of $1 \mathrm{wt} \%$ hydroxyapatite. The lowest flexural strength value was obtained by the addition of $3 \mathrm{wt} \%$ hydroxyapatite. That value increased inversely to $122.95 \pm 11.05 \mathrm{MPa}$ with the addition of $4 \mathrm{wt} \%$ hydroxyapatite content.

Elastic modulus and Vickers hardness of PICNs have shown a similar trend with the addition of hydroxyapatite. With the addition of hydroxyapatite, the elastic modulus of PICN varies from $16.6 \pm 0.6$ to $19.4 \pm 1.9 \mathrm{GPa}$. The elastic modulus of PICN shows an overall increasing trend, with the increasing of hydroxyapatite content from 0 to $4 \mathrm{wt} \%$. The lowest elastic modulus value was achieved at $16.6 \pm 0.6 \mathrm{GPa}$ for PICNs without hydroxyapatite content. Similarly, the maximum elastic modulus value was achieved at 19.4 $\pm 1.9 \mathrm{GPa}$ with the addition of $1 \mathrm{wt} \%$ hydroxyapatite in PICN. The Vickers hardness value varies from $1.12 \pm 0.09$ to $1.31 \pm 0.18 \mathrm{GPa}$. The Vickers hardness of PICN material with hydroxyapatite addition is higher than that of PICN material without adding hydroxyapatite. PICNs without hydroxyapatite show the lowest Vickers hardness $(1.12 \pm 0.09 \mathrm{GPa})$. The Vickers hardness reaches the maximum value of $1.31 \pm 0.18$ $\mathrm{GPa}$ at a hydroxyapatite content of $1 \mathrm{wt} \%$.

The effect of hydroxyapatite on the mechanical properties of PICN is related to the unique structure of this material. The addition of the hydroxyapatite may result in a decrease in the flexural strength, related to the introduced defects. As a kind of hard particle, hydroxyapatite can promote or hinder the crack propagation. The overall effect of hydroxyapatite on flexural strength depends on the dominant role it plays. The presence of nano-hydroxyapatite may serve as a center in PICNs for the formation of defects. Under the action of force, the crack propagates rapidly, resulting in decreasing the flexural strength of PICNs. Hydroxyapatite introduces hard particles into the PICNs. The addition of hydroxyapatite improves the ability of this material to resist deformation which causes the increase in elastic modulus and Vickers hardness. Although the addition of hydroxyapatite may decrease the flexural strength of PICNs and their values can be compared with the commercial CAD/CAM blocks.

The effects of hydroxyapatite and its content on the cell viability and proliferation on PICNs, detected by CCK-8, are shown in Fig. 4. On the 1st day culture process, the PICNs containing hydroxyapatite did not show any promising cell proliferation. On the 3rd day of culture, PICNs without hydroxyapatite and PICNs containing 1-4 wt $\%$ hydroxyapatite have shown significantly different effects of cyto-activity. The cell activity for PICNs containing hydroxyapatite increases 
significantly. On the 5th day of culture, there was no significant variance in cell proliferation of PICNs containing $3 \mathrm{wt} \%$ hydroxyapatite as compared with the 3rd day culture. The optial density (OD) value of 1, 2, 4 $w t \%$ group is significantly lower than that of the 3 rd day. This phenomenon might be related to the growth inhibition of the cells after the proliferation to a certain extent.

Figure 5 shows the morphology of the cellular proliferation of porous materials and PICNs for 4 days. Figures 5(a)-5(e) are the morphologies of rBMSC after 4 days of proliferation for pre-sintered porous ceramics containing $0-4 \mathrm{wt} \%$ hydroxyapatite. It can be seen that rBMSCs are well attached to porous materials, and the porous materials added with hydroxyapatite can promote the adhesion of the cells. It is suggested that the introduction of bioactive substances into porous materials can promote the growth of cells. Figures $5(\mathrm{f})-5(\mathrm{~h})$ represent the morphologies of rBMSC after 4 days of proliferation of PICNs containing 0,1 , and $4 \mathrm{wt} \%$ of hydroxyapatite, respectively. It can be seen that PICNs without hydroxyapatite show poor cell proliferation. PICNs added with 1 and $4 \mathrm{wt} \%$ hydroxyapatite show excellent cell proliferation and attachment, indicating that hydroxyapatite in PICNs can promote the adhesion and spreading of rBMSC. By comparing Figs. 5(a)-5(e) with Figs. 5(f)-5(h), it was found that rBMSCs have a significant proliferation deterioration after polymer infiltration into porous ceramics.

This PICN material without hydroxyapatite has no obvious cytotoxic effect [10]. Biocompatibility can be improved by adding bioactive substances to dental composites. Some researchers have reported the use of

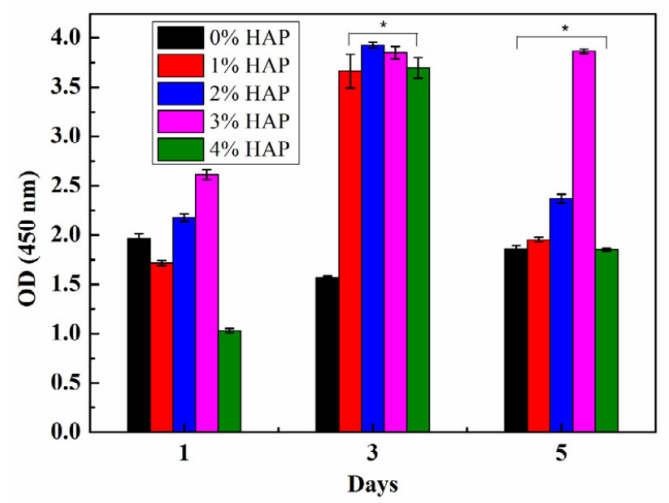

Fig. 4 Viability (absorbance at $450 \mathrm{~nm}$ ) of rBMSCs on the polished surfaces of PICNs assayed via CCK-8 after culture of 1 day, 3 days, and 5 days $(* p>0.05)$.
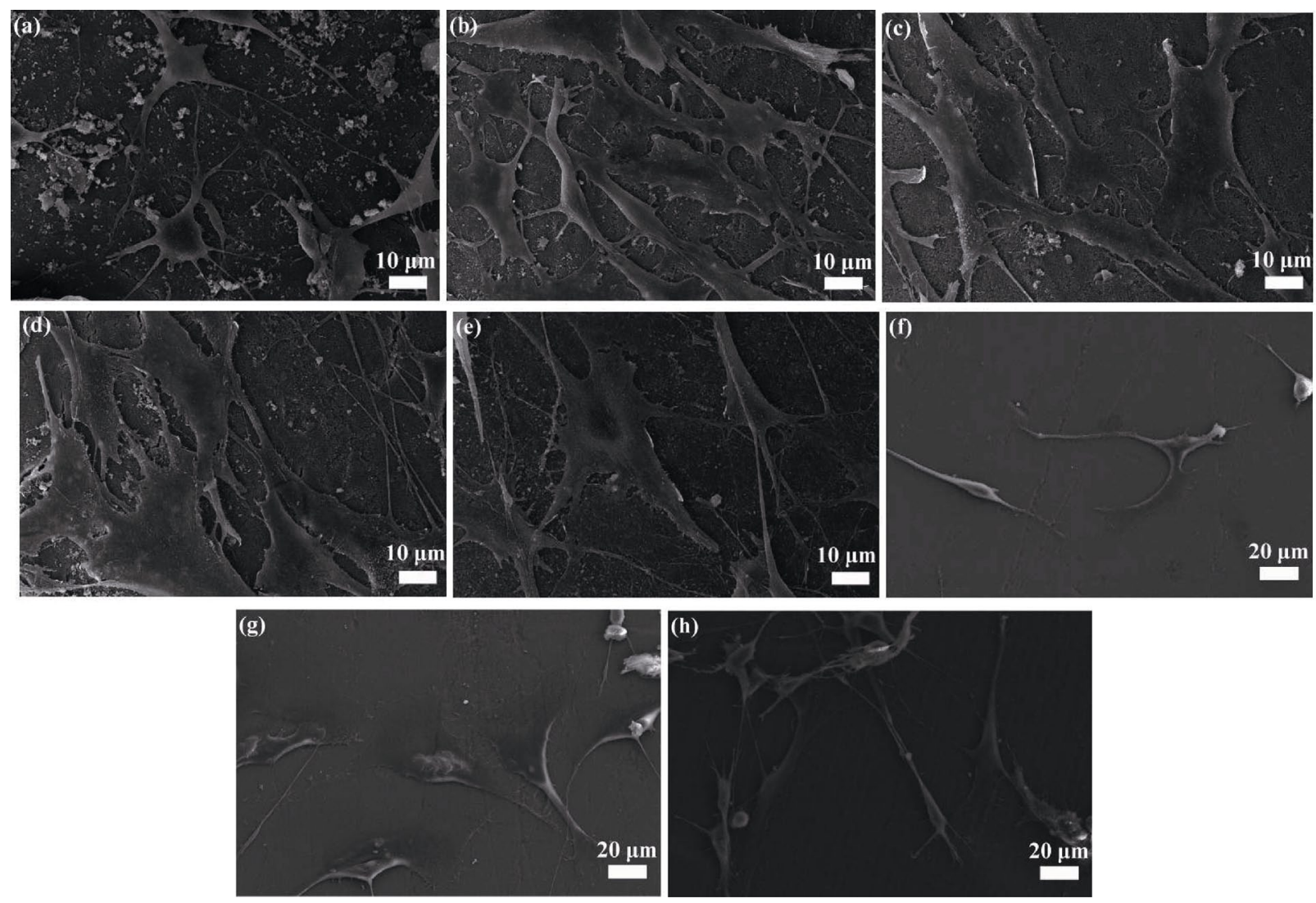

Fig. 5 Proliferation of rBMSCs with porous ceramics and PICN materials after 4 days of proliferation: (a-e) pre-sintered porous ceramics containing $0-4 \mathrm{wt} \%$ hydroxyapatite and ( $\mathrm{f}-\mathrm{h}) \mathrm{PICN}$ with 0,1 , and $4 \mathrm{wt} \%$ hydroxyapatite. 
hydroxyapatite as fillers in dental resin composites [11-14]. The protocol of introducing bioactive substances to PICNs has seldom been found in the literature. As can be seen from Fig. 5, the addition of hydroxyapatite can improve the proliferation and attachment of rBMSCs for both porous ceramics and PICNs. Infiltration of polymer into porous ceramics can cause a decrease of rBMSCs cytoactive. The reason can be explained that the adhesion and spreading of the cell are related to the hydrophily of the material surface. The surface of hydrophilic material is beneficial to the proliferation and attachment of the cell. The methacrylic resin is hydrophobic after curing, so it becomes PICN material after penetrating resin and curing, and hence the surface hydrophobicity of the material increases. It can be found from Figs. 4 and $5(\mathrm{f})-5(\mathrm{~h})$ that the adhesion and spreading of rBMSCs on the surface of the materials can be improved by adding hydroxyapatite to the hydrophobic materials.

\section{Conclusions}

PICN composites have been synthesized. The biocompatibility of PICNs can be improved by the addition of hydroxyapatite nano-powders, and the good mechanical properties can be achieved which are desirable for application in the dental restoration using such PICNs.

\section{Acknowledgements}

This work was financially supported by Beijing Municipal Science \& Technology Commission (No of China. Z171100002017009) and National Natural Science Foundation of China (Grant No. 81671026).

\section{References}

[1] Swain MV, Coldea A, Bilkhair A, et al. Interpenetrating network ceramic-resin composite dental restorative materials. Dent Mater 2016, 32: 34-42.

[2] He LH, Swain M. A novel polymer infiltrated ceramic dental material. Dent Mater 2011, 27: 527-534.

[3] Stawarczyk B, Liebermann A, Eichberger M, et al. Evaluation of mechanical and optical behavior of current esthetic dental restorative CAD/CAM composites. J Mech Behav Biomed Mater 2016, 55: 1-11.
[4] Alharbi A, Ardu S, Bortolotto T, et al. Stain susceptibility of composite and ceramic CAD/CAM blocks versus direct resin composites with different resinous matrices. Odontology 2017, 105: 162-169.

[5] Acar O, Yilmaz B, Altintas SH, et al. Color stainability of CAD/CAM and nanocomposite resin materials. J Prosthet Dent 2016, 115: 71-75.

[6] ISO 4049:2009 Dentistry: Polymer-based restorative materials. Geneva: International Organization for Standardization, 2009.

[7] Oliver WC, Pharr GM. An improved technique for determining hardness and elastic modulus using load and displacement sensing indentation experiments. $J$ Mater Res 1992, 7: 1564-1583.

[8] Cui BC, Li J, Wang HN, et al. Mechanical properties of polymer-infiltrated-ceramic (sodium aluminum silicate) composites for dental restoration. J Dent 2017, 62: 91-97.

[9] Lauvahutanon S, Takahashi H, Shiozawa $\mathrm{M}$, et al. Mechanical properties of composite resin blocks for CAD/CAM. Dent Mater J 2014, 33: 705-710.

[10] Wang HN, Cui BC, Li J, et al. Mechanical properties and biocompatibility of polymer infiltrated sodium aluminum silicate restorative composites. J Adv Ceram 2017, 6: 73-79.

[11] Chen L, Yu QS, Wang Y, et al. BisGMA/TEGDMA dental composite containing high aspect-ratio hydroxyapatite nanofibers. Dent Mater 2011, 27: 1187-1195.

[12] Domingo C, Arcís RW, López-Macipe A, et al. Dental composites reinforced with hydroxyapatite: Mechanical behavior and absorption/elution characteristics. $J$ Biomed Mater Res 2001, 56: 297-305.

[13] Santos C, Luklinska ZB, Clarke RL, et al. Hydroxyapatite as a filler for dental composite materials: mechanical properties and in vitro bioactivity of composites. $J$ Mater Sci: Mater Med 2001, 12: 565-573.

[14] Arcís RW , López-Macipe A, Toledano $\mathrm{M}$, et al. Mechanical properties of visible light-cured resins reinforced with hydroxyapatite for dental restoration. Dent Mater 2002, 18: 49-57.

Open Access This article is licensed under a Creative Commons Attribution 4.0 International License, which permits use, sharing, adaptation, distribution and reproduction in any medium or format, as long as you give appropriate credit to the original author(s) and the source, provide a link to the Creative Commons licence, and indicate if changes were made.

The images or other third party material in this article are included in the article's Creative Commons licence, unless indicated otherwise in a credit line to the material. If material is not included in the article's Creative Commons licence and your intended use is not permitted by statutory regulation or exceeds the permitted use, you will need to obtain permission directly from the copyright holder.

To view a copy of this licence, visit http://createvecommons.org/licenses/by/4.0/. 